\title{
USO DE TESTOSTERONA E SEUS DERIVADOS POR ATLETAS
}

\section{ARTIGO DE REVISÃO}

BORBA, Vinicius José de Andrade ${ }^{1}$

OLIVEIRA, Rodolfo Lee Carvalho de ${ }^{2}$

LIMA, Yuri Correia de ${ }^{3}$

JÚNIOR, Roberto Belo Cardoso ${ }^{4}$

BARROS, Danilo Pontes de Oliveira ${ }^{5}$

BORBA, Vinicius José de Andrade. Et al. Uso de testosterona e seus derivados por atletas. Revista Científica Multidisciplinar Núcleo do Conhecimento. Ano 05, Ed. 08, Vol. 04, pp. 82-95. Agosto de 2020. ISSN: 2448-0959, Link de acesso: https://www.nucleodoconhecimento.com.br/saude/uso-de-testosterona

${ }^{1}$ Discente do curso de graduação em farmácia do Centro Universitário Maurício de Nassau, Recife, Pernambuco, Brasil.

${ }^{2}$ Discente do curso de graduação em farmácia do Centro Universitário Maurício de Nassau, Recife, Pernambuco, Brasil.

${ }^{3}$ Discente do curso de graduação em farmácia do Centro Universitário Maurício de Nassau, Recife, Pernambuco, Brasil.

${ }^{4}$ Discente do curso de graduação em farmácia do Centro Universitário Maurício de Nassau, Recife, Pernambuco, Brasil.

${ }^{5}$ Orientador. Docente do Centro Universitário Maurício de Nassau. Especialista em Citologia Clínica. Citopatologista responsável pelo Controle de Qualidade Interno do Laboratório HEMOLAB diagnósticos. 


\section{RESUMO}

Com a evolução das competições esportivas verificou-se maior busca por substâncias capazes de aprimorar o trabalho corporal e mental a fim de obter maior rendimento nas atividades físicas esportiva. Entre as substâncias usadas por atletas a de maior destaque é a testosterona, pois é o hormônio mais anabólico em relação à síntese de massa muscular. Contribuindo no desenvolvimento de fibras musculares do tipo IIA e IIB, sendo responsável pelo aumento da força, velocidade e aptidão física em geral. Neste sentido, o objetivo dessa pesquisa é relatar o uso de testosterona e seus derivados por atletas e os possíveis danos à saúde. $\mathrm{O}$ artigo dispõe de abordagem qualitativa fundamentada por revisão bibliográfica de caráter narrativo e com finalidade básica estratégica sobre o uso de testosterona e seus derivados por atletas. Foi utilizado base de dados do sítio eletrônico: Lilacs, BVS, Rep. Inst. UNESP, Portal de Revistas - Universidade Católica, IRCCS, SciELO, NLM, MEDLARS composta por 21 artigos e 4 livros. Diante dessa profissionalização esportiva verificouse aumento do uso irracional da testosterona objetivando o destaque diante seus adversários, e com isso seus efeitos colaterais tornaram-se comuns e facilmente observados entre os atletas usuários. Fundamentando então a necessidade de melhor monitoramento dos atletas para uma prevenção primária, podendo diagnosticar, corrigir e punir os atletas usuários de esteroides anabolizantes.

Palavras-chaves: Testosterona, dopagem, efeitos de fármacos.

\section{INTRODUÇÃO}

Com o desenvolvimento das competições esportivas treinadores e atletas buscam ergogênicos exógenos, que são substâncias ou artifícios capazes de melhorar o trabalho corporal e mental, principalmente pela eliminação dos sintomas de astenia, objetivando a melhoria do rendimento nas atividades físicas esportivas competitivas (RUBIO et al, 2010).

Entre os ergogênicos usados pelos atletas, o de maior notoriedade e visibilidade é a testosterona, que é uma substância que apresenta um núcleo derivado da molécula 
do colesterol, portanto tem estrutura lipídica (NIESCHLAGI, 2014). Essa evidência acontece porque a testosterona é o hormônio mais anabólico em relação à síntese de massa muscular, contribuindo no desenvolvimento das fibras musculares tipo IIA e IIB, e também por ser responsável pela diminuição da quantidade de gordura corporal, devido ao aumento do metabolismo corpóreo. Resultando dessa forma no aumento da força, velocidade e aptidão física em geral (PINTO et al, 2017).

A testosterona apresenta fórmula molecular $\mathrm{C}_{19} \mathrm{H}_{28} \mathrm{O}_{2}$, massa molar $288,43 \mathrm{~g} / \mathrm{mol}$ e nomenclatura sistemática 17b-hidróxi-4-androsten-3-um. Seu metabolismo ocorre no fígado, testículo e próstata, a sua meia vida pode variar entre 1 e 24 dias dependendo do tipo de éster, local de sua administração e dose utilizada, já a sua excreção ocorre principalmente pela urina. Sua administração pode ser feita pelas vias: intramuscular, transdérmica, oral e endovenosa em determinados casos (BRUNTON, 2017).

Diante da profissionalização esportiva verifica-se mais investimento, quantidade de patrocínios e oportunidades para os atletas amadores que objetivam viver do seu esporte (ALLAMEH et al, 2016). Havendo então o aumento do uso irracional da testosterona desejando se sobressair diante de seus adversários, e com isso seus efeitos colaterais tornaram-se comuns e facilmente observados entre os atletas. Destacam-se entre eles: Supressão da liberação dos hormônios gonadotróficos, reduzindo assim os níveis do hormônio folículo estimulante, desenvolvimento de acne, calvície, aterosclerose e ainda câncer de próstata (BOFF, 2010).

Diante da capacidade de potencialização da pratica esportiva, o Comitê Olímpico Internacional e a Agência Mundial Antidoping consideram o uso da testosterona e de seus derivados nocivo e fraudulento, devendo o atleta ser penalizado quando rastreado o seu uso (JÚNIOR, 2015)· Por outro lado, é importante analisar e mencionar seu uso clínico prescrito por profissional médico habilitado, como na terapia de reposição de testosterona. Onde estudos mostram que tal medida é adotada com o intuito de suprir a deficiência de testosterona, trazendo benefícios como aumento da densidade óssea, redução de tecido adiposo, ganho de massa magra e melhora geral do bem-estar (BROCH et al, 2014). 
Devido à testosterona ser o hormônio mais usado por atletas, é importante interpretar seu mecanismo de ação, os efeitos positivos e negativos do seu uso assim como de seus derivados. Compreendendo também a importância do seu uso na forma terapêutica clínica prescrita por profissional habilitado. O objetivo dessa pesquisa é relatar o uso de testosterona e seus derivados por atletas e os possíveis danos à saúde.

\section{MÉTODOS}

O artigo dispõe de abordagem qualitativa fundamentada por revisão bibliográfica de caráter narrativo e com finalidade básica estratégica sobre o uso de testosterona e seus derivados por atletas. Foi utilizado base de dados do sítio eletrônico: Literatura Latino-Americana e do Caribe em Ciências da Saúde (Lilacs), Biblioteca Virtual em Saúde (BVS), Repositório Institucional UNESP, Portal de Revistas - Universidade Católica, Scientific Institute for Research, Hospitalization and Healthcare (IRCCS), Scientific Electronic Library Online (SciELO), National Library of Medicine (NLM), Medical Literature Analysis and Retrieval System Online (MEDLARS).

Foram usadas as seguintes palavras-chaves retiradas do site "Descritores em Ciências da Saúde": Metabolismo. Farmacologia. Efeitos Colaterais. Reações Adversas Relacionadas a Medicamentos. Isolamento \& purificação.

A população de estudo do artigo foi composta por 21 artigos e 4 livros com literatura relacionada ao tema de estudo usando material de 2002 a 2020. Quanto à amostra, os artigos foram selecionados a partir da variável de interesse. A seleção foi realizada a partir de leitura de artigos, teses e dissertações encontradas nas bases de dados. Foram incluídas apenas as publicações que responderam à questão do estudo, nos idiomas português, inglês, italiano e espanhol, todos os tipos de delineamentos metodológicos foram aceitos. Diante disso foram construídos quatro eixos temáticos para discussão.

Os aspectos éticos não se aplicam porque a revisão não realizou coleta de dados de seres humanos. 


\section{REFERENCIAL TEÓRICO}

\section{USO DE ESTEROIDES ANABOLIZANTES ENTRE ATLETAS}

Devido à capacidade de melhora da função corporal e mental, eliminação dos sintomas de astenia e melhoria do rendimento geral nas atividades físicas esportivas tornou-se difuso o uso de esteroides anabolizantes (RUBIO, 2010). Um renomado caso dessa prática é a ocorrência do ex-ciclista profissional Lance Edward Gunderson. Atleta nascido nos Estados Unidos em 1971, foi campeão do ciclismo em estrada na Noruega em 1993 e ficou famoso por ser campeão do Tour de France por sete vezes consecutivas (GUTMAN, 2003). Sendo considerado a partir de então, o maior ciclista de todos os tempos (BELL et al, 2016).

Porém, no ano de 2012 mesmo após ter encerrado sua carreira esportiva, perdeu todos os seus títulos obtidos depois do ano 1998 e foi banido do ciclismo competitivo pela União Ciclística Internacional conforme o código da Agência Mundial Antidoping. Pois a Agência Americana Antidoping apresentou um relatório comprovando o uso do hormônio eritropoietina, testosterona e de contínuas transfusões de sangue para melhorar seu desempenho. Posteriormente em janeiro de 2013, Lance Armstrong confessou pela primeira vez o uso de substâncias anabolizantes no programa Oprah Winfrey Show (MAGALHÃES, 2013).

$\mathrm{Na}$ cidade de Rio Claro em São Paulo foi feito um estudo sobre doping e suplementação com onze atletas profissionais e quatro atletas amadores de artes marciais mistas com idade entre 18 e 38 anos no ano de 2015. Foi descoberto que dos quinze atletas entrevistados, todos eles dizem conhecer os efeitos colaterais do uso de anabolizantes (100\%), sendo que 14 deles dizem conhecer um ou mais companheiros de esporte que fazem o uso de esteroides (93,3\%), quatro responderam que já usaram esteroides anabolizantes (26,7\%), sendo todos derivados de testosterona. Revelando quão disseminada essa pratica se encontra na luta, pois 93\% desses atletas têm um ou mais companheiros de treinos que faz uso de anabolizantes (RODRIGUES, 2015). 
Uma pesquisa foi feita em 2018 nas academias de um município do interior do Estado da Bahia, registradas na vigilância sanitária e/ou no Conselho Regional de Educação Física, totalizando quatro academias de musculação. Participaram 100 adultos jovens praticantes de musculação com faixa etária de 18 a 35 anos de ambos os sexos, 50 do sexo masculino e 50 do feminino que se dispusera a participar como voluntários do estudo (OLIVEIRA et al, 2018).

Foi constatado que quase metade dos entrevistados já fez o uso de algum esteroide anabolizante, sendo a testosterona usada pela maioria. Também foi observado que $60,9 \%$ dos usuários relataram sentir efeitos colaterais, sendo a maioria aparecimento de espinha, aumento de libido e alteração na pressão arterial, apenas $8,7 \%$ foram obtidos em farmácia com receita e 2,2\% teve acompanhamento médico. Houve também uma associação entre os usuários: A maioria tem de 18 a 25 anos, é do sexo feminino, tem renda mensal $\leq$ um salário mínimo, $\leq 12$ anos de estudo, é solteira ou divorciada, tem mais de um ano de pratica de musculação e usa/usou menos de 1 mês (OLIVEIRA et al, 2018).

\section{HISTÓRIA DA TESTOSTERONA SINTÉTICA}

Entre os anos de 1803 e 1861 o fisiologista alemão Arnold Adolph Berthold em seus primeiros trabalhos sobre a castração e transplante testicular em aves fez importantes descobertas sobre os hormônios andrógenos, sendo considerado a partir de então o "pioneiro da endocrinologia". Sua pesquisa sobre a ação da molécula de testosterona recebeu apoio em 1889, quando Charles Édouard Brown Séquard, sucessor do famoso fisiologista francês Claude Bernard, auto-injetou o que ele intitulou de "elixir rejuvenescedor", que era administrações subcutâneas de extratos derivados de testículos de cães e de porcos da índia, entre os anos de 1817 e 1894 (KOEHLER, 2017).

$\mathrm{Na}$ revista científica "The Lancef" Charles relatou que seu elixir rejuvenescedor produzia efeitos revigorantes sobre as funções mentais, aumento da agilidade, resistência, energia e força muscular, porém esses efeitos eram transitórios (NIESCHLAGI, 2014). Então junto ao seu assistente Jacques-Arsène d'Arsonval 
iniciou a produção do medicamento e ofereceu esses extratos para seus colegas, a fim de permitir que experimentassem em alguns dos seus pacientes idosos (KOEHLER, 2017).

Já em 1935, Karoly Gyula David e Ernst Laqueur, cientistas financiados pela Organon Company da Holanda, apresentaram um artigo chamado de "Sobre o Hormônio Cristalino Masculino Proveniente dos Testículos e Testosterona" como resultado da sua pesquisa no isolamento da testosterona (KOEHLER, 2017). Porém foi no ano de 1939 que Leopold Stjepan Ruzicka e Adolf Friedrich Johann Butenandt receberam o prêmio Nobel de química por seu trabalho sobre os hormônios sexuais, onde conseguiram produzir sinteticamente a testosterona (HOBERMAN, 2005).

Após a descoberta do poder anabólico da testosterona, fisiculturistas da costa oeste dos Estados Unidos começaram a utilizá-la para aumentar massa muscular e força no ano de 1950, então, seu uso foi disseminado progressivamente entre os atletas dos outros esportes. Esse uso da testosterona também foi observado em atletas em um campeonato de levantamento de peso na capital da Áustria, cidade de Viena no ano de 1954, já objetivando o aumento do rendimento esportivo profissional. Porém seu uso em disputas só foi amplamente divulgado no ano de 1964 nas olimpíadas de Tóquio no Japão no auge da guerra fria, visto que as vitórias esportivas significavam poder político (HOBERMAN, 2005).

A partir do ano de 1970 o Comitê Olímpico Internacional passou a desenvolver campanhas de conscientização e efetivar métodos de testes. Havendo então uma padronização na investigação e rastreio de determinadas substâncias ergogênicas e métodos como manipulação de sangue realizada pelos atletas (NIESCHLAGl, 2014). Banindo das competições aqueles descobertos como sendo usuários destas drogas, com o intuito de orientar e educar os atletas. Porém esse uso é comum até hoje, pois alguns atletas de elite mesmo passando por constantes testes encontram formas de burlá-los (HOBERMAN, 2005). 


\section{SÍNTESE HORMONAL ENDÓGENA DA TESTOSTERONA}

A biossíntese da testosterona processa-se a partir da molécula de colesterol, onde há uma clivagem oxidativa na sua cadeia lateral pela enzima mitocondrial P450 e pelo gene CYP11A1 (NIESCHLAG et al, 2012). Isto faz com que o colesterol perca 6 átomos de carbono, originando a pregnenolona, que é um abundante hormônio cerebral e é precursor da maioria dos hormônios esteroides, sendo considerado a "mãe dos hormônios" (GEBARA et al, 2002).

Posteriormente por atividade da enzima $3 \beta$-hidroxiesteróide desidrogenase será formada a progesterona. Como terceira etapa da síntese endógena a enzima $17 \alpha$ hidroxilase origina a 17a-hidroxiprogesterona. A posteriori sua cadeia lateral será clivada pela 17a-hidroxilase formando a androstenediona (GEBARA et al, 2002). E por último, a enzima 17 $\beta$-hidroxiesteróide desidrogenase reduz o grupamento carbono 17 da androstenediona para dar origem a testosterona, sendo então a etapa de delimitação(MIDZAK et al, 2009).

Outro possível método de formação da testosterona é por ação da 17a-hidroxilase, a partir da 17a-hidroxipregnolona formando a dehidroepiandrosterona, que posteriormente sofre ação da $17 \beta$-hidroxiesteróide desidrogenase e será convertida em androstenediol. Por último, novamente por ação da $3 \beta$-hidroxiesteróide desidrogenase será formada a testosterona. Ambos os mecanismos serão apresentados na figura 1(HANG et al, 2016).

Nos seres humanos, o hormônio testosterona é o andrógeno mais determinante devido a sua potência de caracterização masculina. É secretado principalmente pelo testículo e regulado pelo hormônio luteinizante hipofisário (HANG et al, 2016). Sua produção acontece pelas células de Leydig no interstício dos testículos pelos homens, que está gerenciado pelo hormônio adrenocorticotrófico, a corticotrofina e nos ovários e nas glândulas adrenais das mulheres. Inicia-se logo após o nascimento em níveis muito baixos e atinge seu ápice a partir do período da puberdade, porém diminui acentuadamente após os 35 anos de idade, determinando a andropausa (ROHDEN, 2011). 
Figura 1- Biossíntese da testosterona

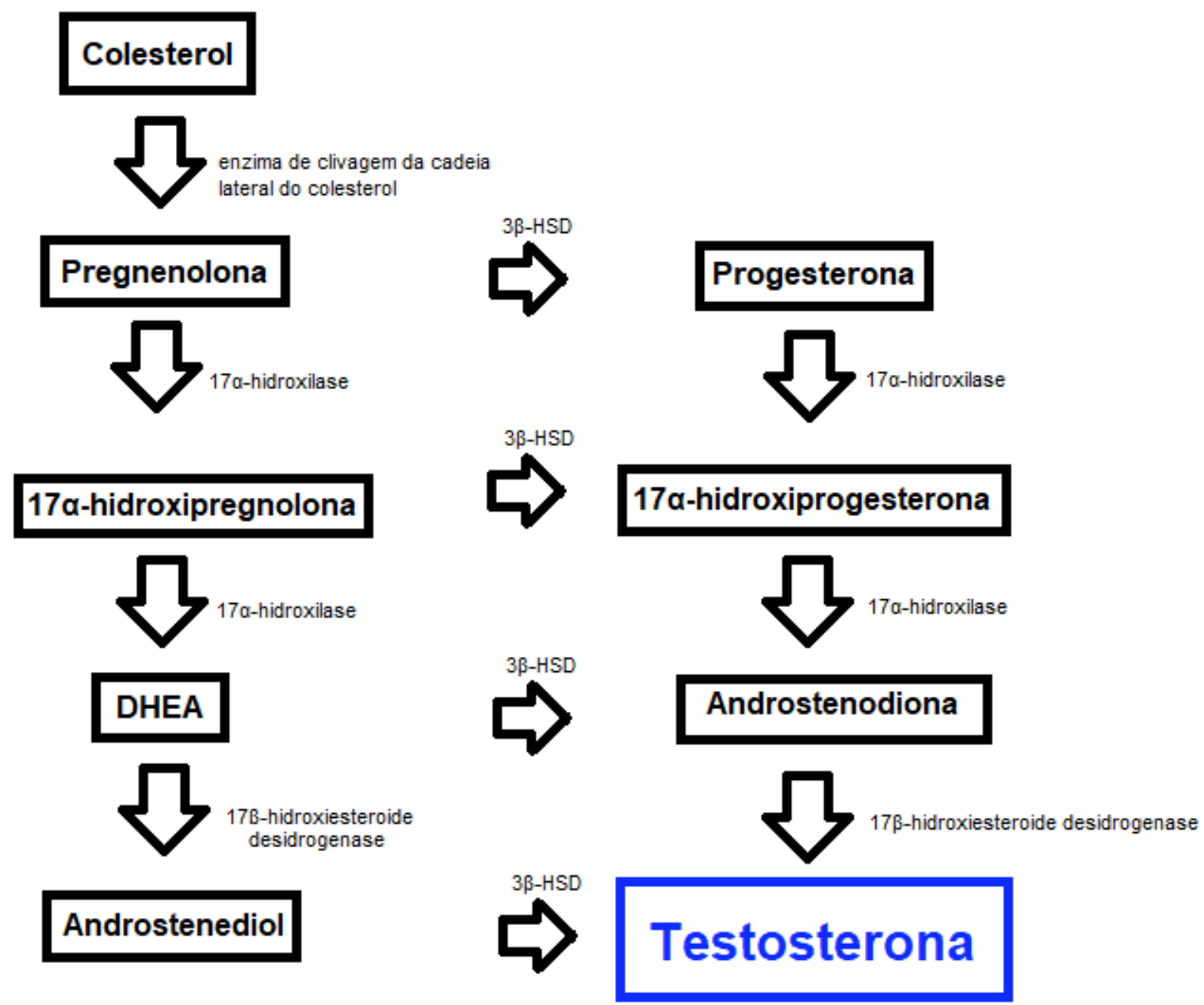

\section{EFEITOS COLATERAIS DO USO DE TESTOSTERONA POR ATLETAS}

A utilização da testosterona de forma inapropriada independente do objetivo pode causar diversas alterações fisiológicas, destacando-se entre elas os seguintes efeitos colaterais: Alterações enzimáticas, fechamento prematuro das epífises ósseas, diminuição da função sistólica e diastólica, aumento nos níveis de lipoproteínas de 
baixa densidade, hipertrofia miocárdica, aumento no volume de placas ateroscleróticas, risco aumentado de desenvolver eventos tromboembólicos arteriais e venosos devido ao aumento na geração de plaquetas e tumores de próstata em geral (ABRAHIN et al, 2013)

Especialmente nos homens é destacado: Supressão da secreção endógena de testosterona, ginecomastia, disúria, atrofia dos testículos devido a um "feedback" negativo que pode resultar em infertilidade e impotência, supressão da liberação dos hormônios gonadotróficos reduzindo os níveis do hormônio folículo estimulante podendo levar a diminuição da contagem, mobilidade e alteração da morfologia dos espermatozoides (MOREIRA et, 2016)

Já nas mulheres destaca-se especialmente o aumento no crescimento de pelos no corpo, aumento da acne através do estimulo das glândulas sebáceas, diminuição no tamanho dos seios, alteração na libido, mudança de voz, menstruação irregular, atrofia ovariana, queda de cabelo e hipertrofia do clitóris (Bl et al, 2018).

Em um estudo publicado na Itália avaliando 600 lutadores profissionais foi insinuada uma relação causal entre justificativa da morte e uso de esteroides anabolizantes. Propondo uma realização de um estudo retrospectivo de 1985 até 2015 para coletar casos de morte prematura extraídos de dados publicados pela empresa americana de mídia e entretenimento “World Wrestling Entertainment” (PROMETTI et al, 2015).

Foi exposto que a expectativa de vida desses atletas usuários é baixa, pois mais $50 \%$ desses indivíduos morrem antes de completarem 50 anos. Foi observado ainda que a morte súbita cardíaca seja responsável por mais da metade dos casos, atribuindo a esse fato uma possível associação com o abuso de androgênicos. Concluindo que é necessário efetivar programas de treinamento respeitando a saúde e também monitorar esses casos, para haver então uma prevenção primária, podendo diagnosticar e corrigir os primeiros sinais de suspeita de abuso de esteroides anabolizantes (PROMETTI et al, 2015). 
Outro estudo realizado em Rondônia em 2019 com praticantes de musculação utilizou como grupo de estudo 189 pessoas com idade entre 18 e 45 anos que foram convidados a fazer a avaliação hepática através da dosagem das enzimas. Foi constatado que nove participantes afirmaram utilizar esteroides anabolizantes para o ganho de massa muscular, sendo que 55,6\% deles não fizeram acompanhamento médico e 33,33\% não tiveram orientação de nenhum profissional da saúde sobre os riscos desse uso. Entre esses nove participantes que afirmaram utilizar esteroides objetivando o aumento de massa muscular $77 \%$ são do sexo masculino e $33 \%$ são do sexo feminino (FREITAS et al, 2019).

Após realização dos exames bioquímicos para a dosagem de enzimas dos usuários, foi observado que houve alterações em algumas dessas enzimas. Somente quatro usuários se submeteram a esses exames bioquímicos, destes, 25\% apresentaram níveis acima dos valores de referência para aspartato aminotransferase, 25\% apresentaram níveis acima dos valores de referência para gama glutamil transferase e 75\% apresentaram níveis acima dos valores de referência para bilirrubina direta (FREITAS et al, 2019).

\section{CONSIDERAÇÕES FINAIS}

Através da análise de literatura pode-se concluir que o uso de testosterona e de seus derivados tem se tornado cada vez mais comum entre atletas. Destacando dessa forma seus efeitos colaterais: Elevação dos valores das enzimas hepáticas, aumento do risco de hipertrofia cardíaca, aumento no volume de placas ateroscleróticas, aumento na geração de plaquetas e tumores de próstata.

Dessa forma foi evidenciada a importância da compreensão sua biossíntese, mecanismos de ação, capacidade de alterações fisiológicas e os tipos de ésteres que determinam sua meia vida plasmática. A fim de haver melhor propagação de informação científica e educação em saúde.

Fundamentando a necessidade de haver melhor monitoramento dos atletas para uma prevenção primária. Podendo diagnosticar, corrigir e punir os atletas desde os 
primeiros sinais de suspeita de abuso de esteroides anabolizantes. Sugerindo então mais pesquisas referentes o tema, pois o uso de esteroides anabolizantes poderá vir a se tornar problema de saúde pública.

\section{REFERÊNCIAS}

ABRAHIN O.S.C; SOUSA E.C. 2013. Páginas 3-8. Disponível em: http://www.scielo.br/scielo.php?pid=S198330832013000400014\&script=sci_arttext

ALLAMEH F; POUR A.M.D.G; BOZORGI A; NEKUIE S; NAMDARI F. 2016. Página 6. Disponível em: <https://www.ncbi.nlm.nih.gov/pmc/articles/PMC4822388/>.

BELL P; HAVE C.T; LAUCHS M. 2016. Página 7. Disponível em: <https://www.sciencedirect.com/science/article/pii/S1756061616300076>.

BI Y; PERRY P.J; ELLERBY M; MURRY D.J. 2018. Páginas 5-12. Disponível em: <https://www.ncbi.nlm.nih.gov/pmc/articles/PMC5915615/>.

BOFF S.R. 2010. Páginas 83-84. Disponível em: $<$ https://bdtd.ucb.br/index.php/RBCM/article/view/1316>.

BROCH D; VARELA Q.D. 2014. Páginas 406-407. Disponível em: <http://ojs.fsg.br/index.php/pesquisaextensao/article/view/2242>

BRUNTON L.L; DANDAN R.H; KNOLLMANN B.C. 2017. Páginas 1760-1761(As Bases Farmacológicas da Terapêutica de Goodman e Gilman).

FREITAS N.C.D; SILVA M.M.R; BASSOLI B.K; SILVA F.C. 2019. Páginas 3-7. Disponível em: <https://periodicos.ufac.br/index.php/SAJEBTT/article/view/2985/2059>.

GEBARA O.C.E; VIEIRA N.W; MEYER J.W; CALICH A.L.G; TAI E.J; PIERRI H; et al. 2002. Páginas 4-8. Disponível em: <http://www.scielo.br/scielo.php?pid=S0066782X2002001500013\&script=sci_arttext\&tlng=pt>. 
GUTMAN B. 2003. Páginas 05-190. Lance Armstrong: A biography.

HANG H.P; RITTER J.M; FLOWER R.J; HENDERSON G. 2016. Páginas 488-497. Rang \& Dale Farmacologia.

HOBERMAN J.M. 2005. Páginas 6-7. Disponível em: <https://www.ncbi.nlm.nih.gov/pmc/articles/PMC1236708/>.

JÚNIOR R.F. 2015. Páginas 14-15. Disponível em: $<$ https://riuni.unisul.br/bitstream/handle/12345/1391/112015_Roosevelt.pdf?sequenc $\mathrm{e}=1$ \&isAllowed $=y>$.

KOEHLER P.J. 2017. Páginas 3-5. Disponível em: < https://worldneurologyonline.com/article/brown-sequard-syndrome-and-networks/>.

MAGALHÃES A.C.S. 2013. Páginas 16-17. Disponível em: $<$ https://attena.ufpe.br/handle/123456789/11791>.

MIDZAK A.S; CHEN H; PAPADOPULOS V; ZIRKIN B.R. 2009. Páginas 5-6. Disponível em: <https://www.sciencedirect.com/science/article/abs/pii/S0303720708003055>.

MOREIRA W.F; SCOSS D.M. 2016. Páginas 8-14. Disponível em: $<$ https://bdtd.ucb.br/index.php/RBCM/article/view/1316>.

NIESCHLAG E; NIESCHLAG $\quad$ S. 2014. Página 7. Disponível em: <https://www.ncbi.nlm.nih.gov/pmc/articles/PMC3955324/ >.

NIESCHLAG E; BEHRE H.M. 2012. Páginas 112-130. Testosterone: Action, Deficiency, Substitution, Cambridge University Press.

OLIVEIRA L.L; NETO J.L.C. 2018. Páginas 7-10. Disponível em: $<$ http://www.scielo.br/scielo.php?script=sci_arttext\&pid=S0101 32892018000300309>. 
PINTO V.C.M; SANTOS P.G.M.D; DANTAS M.P; ARAÚJO J.P.F; CABRAL S.A.T; CABRAL B.G.A.T. 2017. Página 5. Disponível em: $<$ http://pepsic.bvsalud.org/pdf/rbcdh/v27n1/pt_11.pdf>.

PROMETTI P; BELLINI G; AMADDEO P. 2015. Páginas 7-8. Disponível em: $<$ https://moh-it.pure.elsevier.com/en/publications/premature-death-in-professionalwrestlers-cardiovascular-and-neur>.

RUBIO K; NUNES A.V. 2010. Páginas 4-5. Disponível em: $<$ https://portalrevistas.ucb.br/index.php/RBPE/article/download/9289/5550 >.

RODRIGUES G.M. 2015. Páginas 41-45. Disponível em: $<$ https://repositorio.unesp.br/bitstream/handle/11449/136611/000859921.pdf?sequen ce $=1$ \&isAllowed $=y>$.

ROHDEN F. 2011. Páginas 7-8. Disponível em: <http://www.scielo.br/scielo.php?pid=s0104-1832011000100006\&script=sci_arttext>. Enviado: Julho, 2020.

Aprovado: Agosto, 2020. 